\title{
Overexpression of MTA1 inhibits the metastatic ability of ZR-75-30 cells in vitro by promoting MTA2 degradation
}

Long Zhang ${ }^{1} \mathbb{D}$, Qi Wang ${ }^{1}$, Yuzhen Zhou', Qianwen Ouyang ${ }^{6}$, Weixing Dai ${ }^{4,5}$, Jianfeng Chen ${ }^{1,3}$, Peipei Ding ${ }^{1}$, Ling Li ${ }^{3}$, Xin Zhang ${ }^{1}$, Wei Zhang ${ }^{1}$, Xinyue Lv ${ }^{1}$, Luying $\mathrm{Li}^{1}$, Pingzhao Zhang ${ }^{1,3}$, Guoxiang Cai ${ }^{4,5}$ and Weiguo Hu $\mathrm{u}^{1,2,3^{*}}$

\begin{abstract}
Background: As the first member of the metastasis-associated protein (MTA) family, MTA1 and another MTA family member, MTA2, have both been reported to promote breast cancer progression and metastasis. However, the difference and relationship between MTA1 and MTA2 have not been fully elucidated.

Methods: Transwell assays were used to assess the roles of MTA1 and MTA2 in the metastasis of ZR-75-30 luminal B breast cancer cells in vitro. Immunoblotting and qRT-PCR were used to evaluate the effect of MTA1 overexpression on MTA2. Proteases that cleave MTA2 were predicted using an online web server. The role of neutrophil elastase (NE) in MTA1 overexpression-induced MTA2 downregulation was confirmed by specific inhibitor treatment, knockdown, overexpression and immunocytochemistry, and NE cleavage sites in MTA2 were confirmed by MTA2 truncation and mutation. The effect of MTA1 overexpression on the intrinsic inhibitor of NE, elafin, was detected by qRT-PCR, immunoblotting and treatment with inhibitors.

Results: MTA1 overexpression inhibited, while MTA2 promoted the metastasis of ZR-75-30 cells in vitro. MTA1 overexpression downregulated MTA2 expression at the protein level rather than the mRNA level. NE was predicted to cleave MTA2 and was responsible for MTA1 overexpression-induced MTA2 degradation. NE was found to cleave MTA2 in the C-terminus at the 486, 497, 542, 583 and 621 sites. MTA1 overexpression activated NE by downregulating elafin in a histone deacetylase- and DNA methyltransferase-dependent manner.

Conclusions: MTA1 and MTA2 play opposing roles in the metastasis of ZR-75-30 luminal B breast cancer cells in vitro. MTA1 downregulates MTA2 at the protein level by epigenetically repressing the expression of elafin and releasing the inhibition of neutrophil elastase, which cleaves MTA2 in the C-terminus at multiple specific sites.
\end{abstract}

Keywords: MTA1, MTA2, Neutrophil elastase, Elafin, Breast cancer metastasis

\section{Background}

Breast cancer is the most prevalent cancer women suffer from worldwide and is estimated to be the cancer with the highest morbidity and second highest mortality among women in the United States [1]. Breast cancer is a highly heterogeneous cancer; therefore, it is classified

\footnotetext{
* Correspondence: weiguohu@fudan.edu.cn

${ }^{1}$ Fudan University Shanghai Cancer Center and Institutes of Biomedical Sciences, Collaborative Innovation Center of Cancer Medicine, Shanghai Medical College, Fudan University, 270 Dong'an Road, Shanghai 200032, China

2Department of Immunology, Shanghai Medical College, Fudan University, 130 Dong'an Road, Shanghai 200032, China

Full list of author information is available at the end of the article
}

into different subtypes, which include normal breast, basal-like, claudin-low, HER2 positive (HER2+), luminal $\mathrm{A}$ and luminal $\mathrm{B}$ cancers, according to the histological features and molecular traits of the cancer $[2,3]$. Luminal B breast cancer shows more aggressive phenotypes than luminal A breast cancer and higher insensitivity to neoadjuvant chemotherapy than basal-like and HER2+ breast cancers, leading to its obstinacy and poor prognosis of patients [2, 4]. In addition, luminal B breast cancer tends to metastasize, increasing the mortality of luminal B breast cancer patients $[2,5]$. In fact, breast cancer metastasis is the major lethal factor that affects patient mortality [6].

(C) The Author(s). 2019 Open Access This article is distributed under the terms of the Creative Commons Attribution 4.0 International License (http://creativecommons.org/licenses/by/4.0/), which permits unrestricted use, distribution, and 
To overcome the problem of breast cancer metastasis, a 13762NF rat mammary adenocarcinoma metastasis model was established, and the first member of the metastasis associated protein (MTA) family, MTA1, was discovered by differential cDNA library screening in 1994 [7]. The other two members of the MTA family, MTA2 and MTA3, were identified afterwards [8, 9]. Subsequent studies have revealed that MTA1, MTA2 and MTA3 are constitutive components of nucleosome remodeling and histone deacetylase (NuRD) complex [10-12]. The NuRD complex possesses multiple activities, such as histone deacetylase activities and methyl-CpG-binding-related activities, because of the composition of histone deacetylase $1 / 2$ (HDAC1/2) and methyl-CpG-binding domain protein 2/3 (MBD2/3), respectively $[10,13]$. MTA1 and MTA2 have both been reported to be pivotal for epithelial-mesenchymal transition (EMT) and metastasis of breast cancer, while MTA3 has been reported to inhibit EMT [12, 14-16]. EMT is a critical step among a sequence of discrete steps that cancer cells undergo to achieve metastasis $[17,18]$. During EMT, the EMT-inducing factors Snail, Slug, TWIST and ZEB1 are upregulated, the epithelial molecule E-cadherin is downregulated and the mesenchymal molecule $\mathrm{N}$-cadherin is upregulated $[17,18]$.

Both being potent breast cancer metastasis-promoting factors of the MTA family, the difference and relationship between MTA1 and MTA2 remain an enigma because of insufficient investigations [19]. In this study, we aimed to examine the different roles that MTA1 and MTA2 may play in breast cancer metastasis and investigate their relationship. We investigated the roles of MTA1 and MTA2 in the metastasis of the ZR-75-30 luminal B breast cancer cell line [20, 21], and we found that the overexpression of MTA1 inhibits the metastasis of ZR-75-30 cells by downregulating MTA2, which has not been clarified previously [19]. Mechanistically, the overexpression of MTA1 transcriptionally downregulated the intrinsic inhibitor of the neutrophil elastase (NE), elafin, to promote the degradation of MTA2 that was mediated by NE, therefore inhibiting the metastasis of ZR-75-30 cells in vitro. Overall, our study provided direct evidence for the different roles that MTA1 and MTA2 play in breast cancer cell metastasis and clarified the mechanism by which MTA1 downregulates MTA2 at the protein level.

\section{Methods}

\section{Cell culture and stable cell line establishment}

293FT cells (Thermo Fisher Scientific) were cultured in Dulbecco's Modified Eagles Medium supplemented with $10 \%$ fetal bovine serum (FBS) and 1\% antibiotics (penicililin and streptomycin) HeLa cells (Type Culture Collection Cell Bank, Chinese Academy of Sciences) and ZR-75-30 cells (a kind gift from the Department of Breast Surgery,
Fudan University Shanghai Cancer Center) were cultured in RPMI 1640 medium containing 10\% FBS and 1\% antibiotics. MDA-BM-231 cells (a kind gift from the Department of Breast Surgery, Fudan University Shanghai Cancer Center) were cultured in Leibovitz's L-15 medium supplemented with $10 \%$ FBS and $1 \%$ antibiotics. All cells were maintained at $37^{\circ} \mathrm{C}$ in a humidifed atmosphere containing $5 \% \mathrm{CO}_{2}$ in a cell incubator (Thermo Fisher Scientific). ZR-75-30-Vector/MTA1/MTA2 stable cell lines were established by infection of lenti-viruses and selection by flow cytometry on MoFlo ${ }^{\mathrm{Tm}}$ XDP (Beckman Coulter). ZR-75-30-shMTA2-NC/\#1/\#2, ZR-75-30-shElafin-NC/\#1/ $\# 2$ were established by infection of lenti-viruses and selection by puromycin. ZR-75-30-MTA1-shNE-NC/\#1/\#2 stable cell lines were established on the basis of ZR-75-30-MTA1 by infection of lenti-viruses and selection by puromycin.

\section{Antibodies and reagents}

Anti- $\beta$-Actin antibody (sc-47,778), anti-MTA1 antibody (sc-17,773) and anti-MTA2 antibody (sc-55,566) were purchased from Santa Cruz Biotechnology (Dallas, TX, USA). Anti-Neutrophil Elastase antibody (ab131260) and anti-Elafin/Skalp antibody (ab46774) were purchased from Abcam (Cambridge, MA, USA). HA-Tag (C29F4) Rabbit $\mathrm{mAb}$ (\#3724) was purchased from Cell signaling technology (Beverly, MA, USA). Monoclonal ANTI-FLAG ${ }^{\circ}$ 2 antibody (F3165) was purchased from Merck Sigma company (Burlington, MA, USA). Anti-DDDDK-tag mAb (M185-3 L) was purchased from MBL International Corporation (Woburn, MA, USA). HRP conjugated secondary antibodies Goat anti-mouse IgG $(\mathrm{H}+\mathrm{L})$, HRP conjugate (SA00001-1) and Goat anti-rabbit IgG $(\mathrm{H}+\mathrm{L})$, HRP conjugate (SA00001-2) were purchased from Proteintech Group (Rosemont, IL, USA). Fluorescent secondary antibodies Goat anti-Rabbit IgG ( $\mathrm{H}+\mathrm{L})$ Highly Cross-Adsorbed Secondary Antibody, Alexa Fluor 488 (A-11034) and Goat anti-Mouse IgG (H+L) Highly Cross-Adsorbed Secondary Antibody, Alexa Fluor 594 (A-11032) and lipofectamine 3000 (L3000015) were purchased from Thermo Fisher Scientific (Waltham, MA, USA). Puromycin (P8230) was purchased from Solarbio biotechnology (Shanghai, China). Cycloheximide (CHX, HY-12320) was purchased from MedChemExpress (Monmouth Junction, NJ, USA). Histone deacetylases (HDAC) inhibitor suberoylanilide hydroxamic acid (SAHA, also known as Vorinostat, MK0683; S1047), Sivelestat (S8136), MG132 (S2619) and Azacitidine (AZA, S1782) were purchased from Selleck Chemicals (Shanghai, China).

\section{Plasmids}

To construct core plasmids overexpressing MTA1/ MTA2, CDSs of MTA1/MTA2 were inserted into pCDH-EF1-MCS-T2A-copGFP. shRNA plasmids targeting 
MTA2/NE/Elafin were purchased from Shanghai Genechem. To construct FLAG-tagged MTA1/NE over-expressing plasmids, the CDS of MTA1/NE (without stop codon) were subcloned into pCMV-C-FLAG vector. To construct HA-tagged MTA2 and HA-tagged MTA2 truncated $\mathrm{N}$-terminal and $\mathrm{C}$-terminal mutants, specific primers were designed to subclone corresponding sequences from MTA2 (without stop codon) into pCMV-C-HA vector. For site mutation, specific mutation primers were designed and corresponding plasmids were mutated according to the manufacture of KOD-Plus-Mutagenesis Kit (SMK-101, TOYOBO, Japan). Mutations were confirmed by sequencing the plasmids. All the primers used to construct truncated mutants and point mutations are in Additional file 1: Table S1.

\section{Lentiviral particles production}

To generate lentiviruses, core plasmids that overexpress or knockdown genes were co-transfected into 293FT cells with packaging plasmid psPAX2 and envelope plasmid pMD2.G at a ratio of 4:3:1 by Neofect ${ }^{\text {ti }}$ DNA transfection reagent (TF201201, Neofect biotech, Beijing, China). After $48 \mathrm{~h}$ of incubation, lentiviral particles were harvested.

\section{Transfection}

To detect the effect of MTA1 expression on the migration and invasion of MDA-MB-231 cells, MDA-MB-231 cells were transfected with pCDH-EF1-MCS-T2AcopGFP-MTA1 or empty vector as a control by lipofectamine 3000 as the manual instructed and cells were seeded for transwell assays after $36 \mathrm{~h}$ of transfection. After $48 \mathrm{~h}$, cells were harvested for immunoblotting. To detect the effect of overexpression of MTA1 to MTA2 and its mutants, pCMV-C-FLAG-MTA1 and corresponding plasmids that overexpress MTA2 mutants were co-transfected into 293FT cells by Neofect ${ }^{\text {ti }}$ DNA transfection reagent. For instance, $1250 \mathrm{ng}$ pCMV-C-FLAG empty vector and $1250 \mathrm{ng}$ pCMV-C-HA-MTA2 were co-transfected to 293FT cells seeded in a well of 6 well plate as the control group and $1250 \mathrm{ng}$ pCMV-C-FLAGMTA1 and 1250 ng PCMV-C-HA-MTA2 were co-transfected in the same as the experimental group. After $48 \mathrm{~h}$ of incubation, transfected cells were harvested for immunoblot. Simlar transfections were conducted for other cells.

\section{Immunoblot}

To detect the levels of proteins in stable cell lines, the same amount of stable control cells and stable overexpression/knockdown cells were seeded in wells of 6 well plates. After $24 \mathrm{~h}$ incubation, cells were lysed by $1.25 \times$ SDS loading buffer containing 1\% $\beta$-mercaptoethanol. For transfected 293FT cells, after transfection, cells were incubated for $48 \mathrm{~h}$, and then were lysed as above. Cell lysates were then heated in a heating block for $10 \mathrm{~min}$ at $100^{\circ} \mathrm{C}$ with vigorous shaking. Same amount of heated cell lysates was loaded for SDS-PAGE respectively, followed by being transferred to PVDF membranes. Membranes were washed for 3 times with TBST and then blocked with $5 \%$ non-fat milk diluted with TBST at room temperature for $2 \mathrm{~h}$. Primary antibodies diluted with 5\% non-fat milk/TBST were incubated with blocked membranes for $2 \mathrm{~h}$ at room temperature or overnight at $4{ }^{\circ} \mathrm{C}$ on a gentle shaking platform. Membranes were washed with TBST for 3 times and then incubated with secondary antibodies conjugated with HRP diluted with $5 \%$ non-fat milk/TBST for $45 \mathrm{~min}$ at room temperature. Finally, membrane was washed by TBST for 3 times and developed with Immobilon Western Chemiluminescent HRP Substrate (WBKLS0500, MerckMillipore, (Burlington, MA, USA)). Images were captured with ImageQuant ${ }^{\text {tm }}$ LAS 4000 biomolecular imager (GE Healthcare).

\section{Protein stability assay}

To validate the effect of MTA1 to the stability of MTA2 protein, ZR-75-30-Vector and ZR-75-30-MTA1 cells were seeded and treated with Cycloheximide (CHX) at a final concentration of $20 \mu \mathrm{M}$ for $0,10,25,90,240,360$ min respectively. Then cells were harvested followed by immunoblot detection.

\section{Quantitative RT-PCR (qRT-PCR)}

Total RNA was extracted from cells as the manufacture of TRIzol reagent (10296010, Thermo Fisher Scientific (Waltham, MA, USA)) indicated. RNAs were reversely transcribed into cDNA using PrimeScript ${ }^{\mathrm{Tw}}$ RT Master Mix (RR036A, Takara Bio Inc. (Japan)). qRT-PCR assay was conducted using SYBR ${ }^{\circ}$ Premix Ex Taq ${ }^{\mathrm{mi}}$ II (Tli RNaseH Plus) (RR820Q, Takara Bio Inc.(Japan)) on a QuantStudio ${ }^{\text {Tx }}$ 7 Flex Real-Time PCR System platform (Thermo Fisher Scientific (Waltham, MA, USA)) following standard protocols. The expression of $\beta$-actin was set as endogenous control and the expressions of genes were calibrated to that of corresponding control cells. Data analyses were conducted by QuantStudio Real-Time PCR Software. RQ values (relative quantified value of mRNA expression) were calculated by the same software followed by two-tailed Student's t test to determine significant differences between two groups. $P<0.05$ was considered statistically significant. All the primers designed for qRT-PCR are in Additional file 1: Table S1.

\section{Immunocytochemistry}

HeLa cells were seeded on the sterilized round coverslips that were inserted in to a well of a 24 well plate. After $12 \mathrm{~h}$ growth, cells were transfected with pCMV-C-HA-MTA2 + 
pCMV-C-FLAG plasmids or pCMV-C-HA-MTA2 + pCMV-C-FLAG-NE plasmids by Neofect reagent. Similar transfections were conducted for other cells. After $48 \mathrm{~h}$ of transfection, cells were fixed with $4 \%$ paraformaldehyde at room temperature for $30 \mathrm{~min}$. Cells were then permeabilized on ice with $0.2 \%$ Triton X-100/PBS for $5 \mathrm{~min}$, followed by washing 3 times using PBS. Then cells were blocked with $1 \% \mathrm{BSA} / \mathrm{PBS}$ at room temperature for $1.5 \mathrm{~h}$ and primary antibodies diluted with $1 \%$ BSA/PBS were applied for an incubation with cells at room temperature for $2 \mathrm{~h}$. Cells were then washed by PBS for 3 times to wash off unbounded antibodies and incubated with fluorescent secondary antibodies at room temperature for $1.5 \mathrm{~h}$. Then cells were washed and the coverslips were mounted with mounting medium containing DAPI. After an incubation at $4{ }^{\circ} \mathrm{C}$ for $15 \mathrm{~min}$, slides were observed and images were captured at Leica TCS-SP5 confocal system.

\section{Cell migration and invasion assays}

For cell migration assay, cells were harvested, washed by PBS for twice, resuspended by DMEM medium without FBS and counted by Vi-CELL XR (Beckman Coulter). Transwell ( $8 \mu \mathrm{m}, 353097$, Corning) chambers were inserted to corresponding wells in 24 well plate that already contain $500 \mu \mathrm{L}$ DMEM with $10 \%$ FBS. For stable cell lines established on ZR-75-30 cells, without noticing, $2 \times 10^{4}$ cells were added to the upper chamber and incubated for $24 \mathrm{~h}$. For cell invasion assays, $60 \mu \mathrm{L}$ matrigel (Matrige ${ }^{\mathrm{Tm}}$ Basement Membrane Matirx, 356,234, BD (Franklin Lakes, NJ, USA)) diluted with DMEM at a ratio of 1:10 was added to the upper chamber. The set was balanced at $4{ }^{\circ} \mathrm{C}$ for 30 min and then moved into cell incubator overnight to dry the gel. Similar with cell migration assay, cells were harvested, washed, resuspended and counted. $4 \times 10^{4}$ cells were added to the matrigel precoated upper chamber under which the well contains $500 \mu \mathrm{L}$ DMEM with $10 \%$ FBS, and incubated for $48 \mathrm{~h}$. For migration and invasion assays performed on MDA-MB-231 cells or ZR-75-30-MTA1 cells, after $36 \mathrm{~h}$ of transfection, $4 \times 10^{4}$ and $8 \times 10^{4}$ cells were added to the upper chamber and incubated for $24 \mathrm{~h}$ and $48 \mathrm{~h}$ respectively. For both cell migration assay and invasion assay, after incubation, medium of the upper chambers were discarded and cells migrated to the lower chamber were fixed with $4 \%$ paraformaldehyde at room temperature for $30 \mathrm{~min}$, stained with $0.5 \%$ crystal violet at room temperature for $30 \mathrm{~min}$, and washed by PBS for 3 times. Cells remained on the upper chamber were erased gently with a cotton-tipped swap. Cells were observed and images were captured at an Olympus microscope system. Migrated and invaded cells were then counted in randomly chosen three out of eight equally divided areas, followed by two-tailed Student's $t$ test to determine significant differences between two groups. $P<0.05$ was considered statistically significant.

\section{Luminal B breast cancer tissue samples}

Twenty paraffin-embedded tissue samples were derived from 20 luminal B breast cancer patients diagnosed and treated at the Department of Breast Surgery, The Third Hospital of Nanchang, Jiangxi Province, China. The Ethics Committee at The Third Hospital of Nanchang approved the utilization of samples, and all patients signed the informed consent form.

\section{Immunohistochemistry (IHC) staining assay}

To detect the protein level of MTA1 and MTA2 in luminal B breast cancer patients, IHC was performed. Briefly, slices were deparaffinized in xylol. $10 \mathrm{mM}$ sodium citrate ( $\mathrm{pH}$ 6.0) was used for antigen retrieval, and endogenous peroxidase activity was eliminate with $3 \%$ $\mathrm{H}_{2} \mathrm{O}_{2}$. Then the slices were blocked with $1 \%$ bovine serum albumin/phosphate-buffered saline. Slices were incubated with anti-MTA1 antibody (sc-17,773, 1:100) or anti-MTA2 antibody (sc-55,566, 1:100) and placed in a humidified chamber and at $4{ }^{\circ} \mathrm{C}$ overnight. Reactions were developed using GTvision TM III (Gene Technology, Shanghai, China) and counterstained with 10\% hematoxylin. At last, the stained slices were dehydrated and mounted with resinene. The staining indexes (0-12) for MTA1/MTA2 was obtained as the staining intensity (negative (0); weak (1); moderate (2); and strong (3)) multiplied by the proportion of positive staining $((0-$ $25 \%$ (1); $25-50 \%$ (2); $50-75 \%$ (3); and $75-100 \%(4))$.

\section{Results}

MTA1 overexpression inhibited the metastasis of ZR-7530 luminal $B$ breast cancer cells in vitro

To investigate the role of MTA1 in luminal B breast cancer metastasis, we first established two stable cell lines, ZR-75-30-MTA1 overexpressing MTA1 and ZR75-30-Vector as a control. MTA1 overexpression was confirmed by immunoblotting (Fig. 1a). Then, we evaluated the metastatic potential of these cell lines by Transwell chamber cell migration and invasion assays. Surprisingly, contradictory to the well-established roles of MTA1, the overexpression of MTA1 significantly inhibited the migration and invasion of ZR-75-30 cells in vitro (Fig. $1 \mathrm{~b}$ and $\mathrm{c}$ ).

We hypothesized that the metastasis-inhibitory role of MTA1 in ZR-75-30 cells may due to the particularity of ZR-75-30 cell line. To testify our hypothesis, we overexpressed MTA1 in MDA-MB-231 cells, a triple negative breast cancer cell line (Fig. 1d). The results of migration and invasion assays indicated that overexpression of MTA1 significantly promoted the migration and invasion of MDA-MB-231 cells in vitro (Fig. 1e and f). The results showed that MTA1 played different roles in the migration and invasion of different breast cancer cells in vitro. 


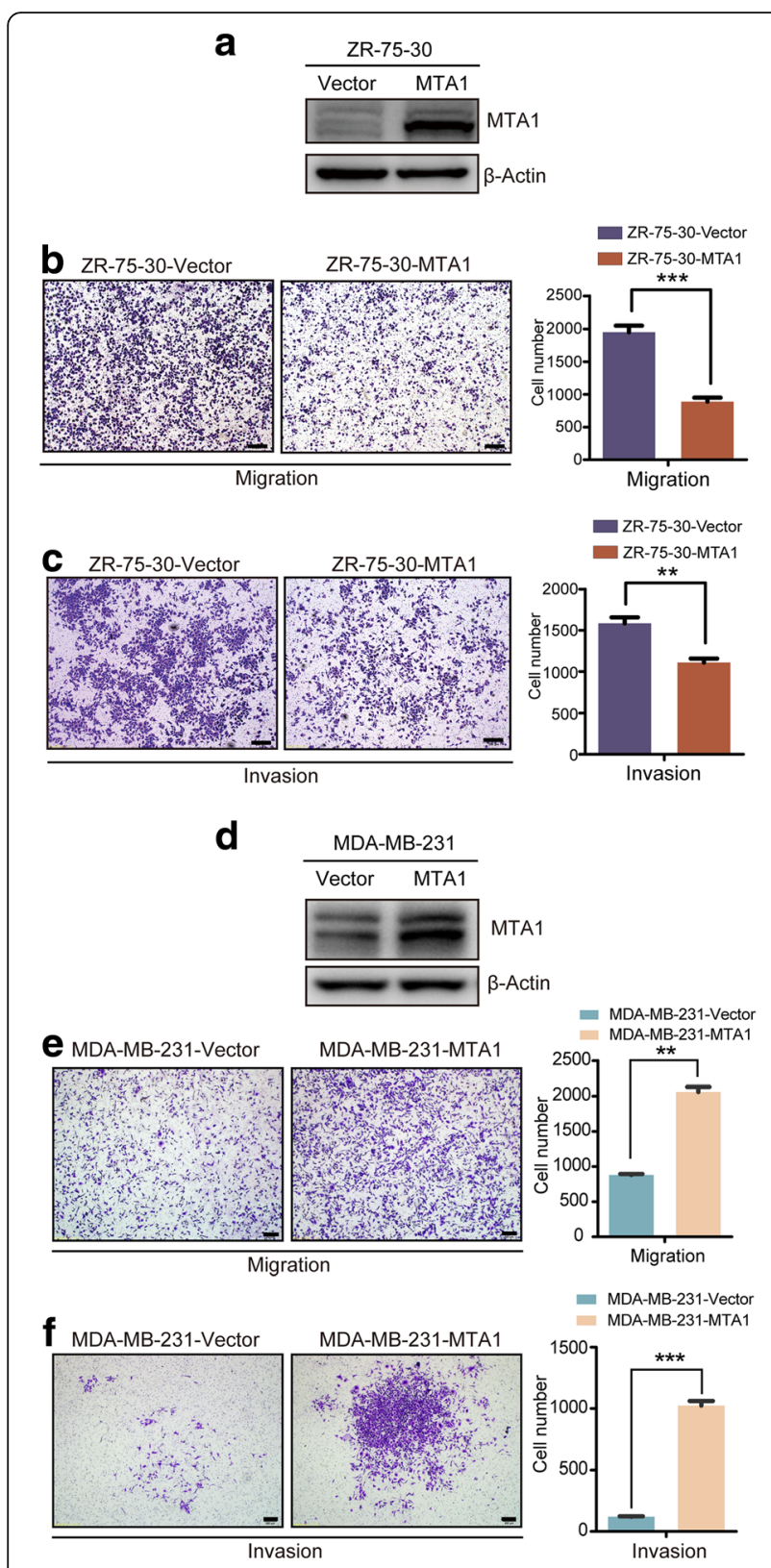

Fig. 1 MTA1 overexpression inhibited the metastasis of ZR-75-30 cells. a MTA1 was overexpressed in ZR-75-30-MTA1 stable cell line compared to its control cell line ZR-75-30-Vector. Detected by immunoblot. $\mathbf{b}$ Overexpression of MTA1 significantly inhibited the migration and (c) invasion of ZR-75-30 cells in vitro. Detected by transwell migration and invasion assays. 30,000 cells migrated for 24 h. Scale bars $=200 \mu \mathrm{m}$, mean $\pm \mathrm{SD}, n=3$, ${ }^{* *} P<0.001$, ${ }^{* *} P<0.01$, $\mathrm{t}$ test. c MTA1 was overexpressed in MDA-MB-231 cells compared with control cells. $\mathbf{d}$ Overexpression of MTA1 significantly promoted the migration and (e) invasion of MDA-MB-231 cells in vitro. Scale bars $=200 \mu \mathrm{m}$, mean $\pm \mathrm{SD}, n=3,{ }^{* * *} P<0.001,{ }^{* *} P<0.01$, $\mathrm{t}$ test

\section{Overexpression of MTA1 downregulated MTA2, which promoted the metastasis of ZR-75-30 cells}

The overexpression of MTA1 has been shown to downregulate the expression of MTA2 at the protein level instead of by transcriptional repression in breast cancer cells [19]. Because of the role of MTA2 in breast cancer metastasis, we hypothesized it's the same reason for MTA1-induced metastasis inhibition. To verify our hypothesis, we evaluated the protein expression level of MTA2 in ZR-75-30-MTA1 cells by immunoblotting and found that it decreased (Fig. 2a).

To validate the role of MTA2 in ZR-75-30 metastasis, we established a ZR-75-30-MTA2 cell line using the same system used to establish the ZR-75-30-MTA1 cell line (Fig. 2b). MTA2 overexpression significantly enhanced ZR-75-30 cell metastasis in vitro (Fig. 2c and d). Knocking down MTA2 using two shRNAs targeting MTA2 significantly inhibited the metastasis of ZR-75-30 cells in vitro (Fig. 2e and f).

To further verify our hypothesis, we measured the mRNA expression level of MTA2 in ZR-75-30-Vector and ZR-75-30-MTA1 cells by qRT-PCR. In accordance with previous studies, the overexpression of MTA1 did not repress MTA2 transcription (Fig. 2g).To further determine if there's negative correlation between the protein levels of MTA1 and MTA2 in luminal B breast cancer patients, we performed Immunohistochemistry (IHC) staining for MTA1 and MTA2 on slices prepared from serial section. Scoring of the IHC staining of MTA1 and MTA2 indicated a negative correlation between the protein level of MTA1 and MTA2 in luminal $\mathrm{B}$ breast cancer tissues $(P<0.05)$, and representative images are shown (Fig. 2h).

Both MTA1 and MTA2 have been reported to promote EMT and breast cancer metastasis. To investigate whether the different roles of MTA1 and MTA2 in ZR-75-30 cell metastasis are caused by the different roles of these factors in the EMT of ZR-75-30 cells, we evaluated the expression levels of the indicators of EMT (Snail and N-cadherin) and found that both MTA1 and MTA2 upregulated the expression of these indicators (Fig. 2i).

These data indicated that although both MTA1 and MTA2 promoted the EMT process in ZR-75-30 cells, the overexpression of MTA1 inhibited the metastasis of ZR-75-30 cells by decreasing MTA2 protein stability.

\section{Neutrophil elastase is responsible for MTA1-induced MTA2 degradation}

To further confirm the influence of MTA1 on the stability of MTA2, we conducted a protein stability assay using cycloheximide $(\mathrm{CHX})$. The results showed that the overexpression of MTA1 significantly decreased the stability of MTA2 (Fig. 3a).To elucidate the mechanism by which MTA1 downregulates MTA2, we first treated ZR-75-30-MTA1 cells with MG132 (5 $\mu \mathrm{M}$ for $12 \mathrm{~h})$ and detected an upregulation of MTA1 (Fig. 3b), which was reported to be regulated by the ubiquitin-proteasome 


\section{a}

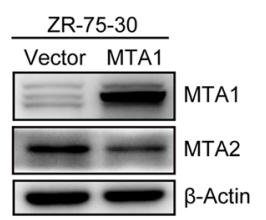

b

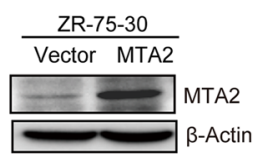

e ZR-75-30-shMTA2
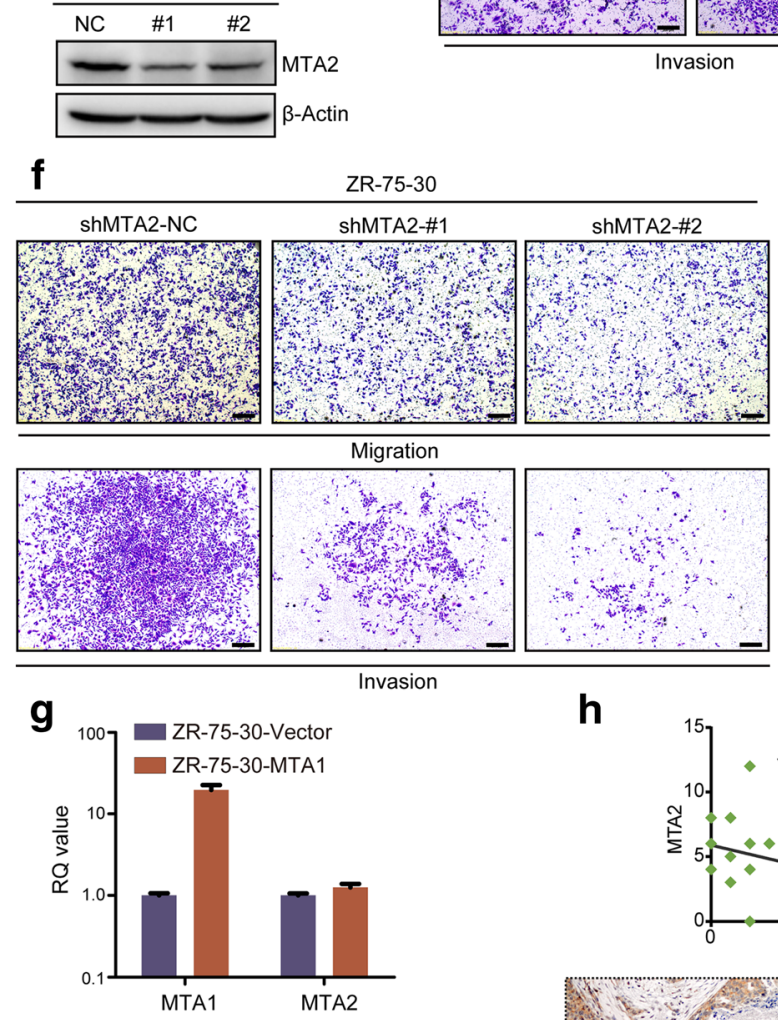

i

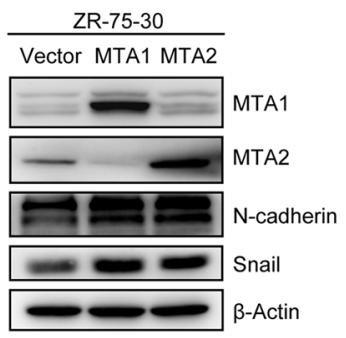

h

莫,

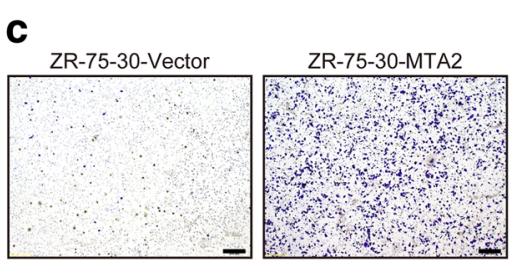

d

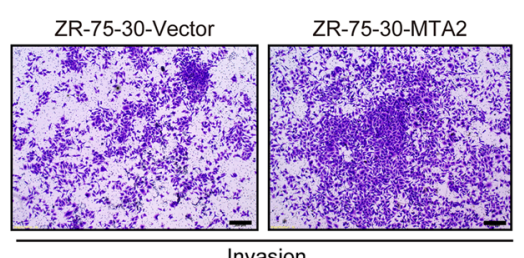

nvasion

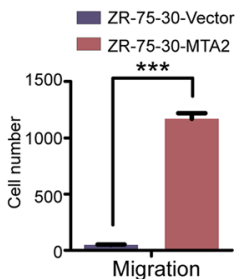

Migration

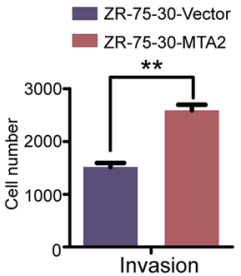

Invasion

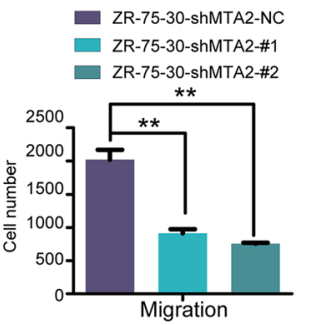

ZR-75-30-shMTA2-NC

ZR-75-30-shMTA2\#1

ZR-75-30-shMTA2-\#2

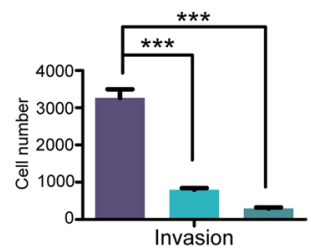

$Y=-0.3538^{*} X+5.886$

$10-\quad P<0.05$
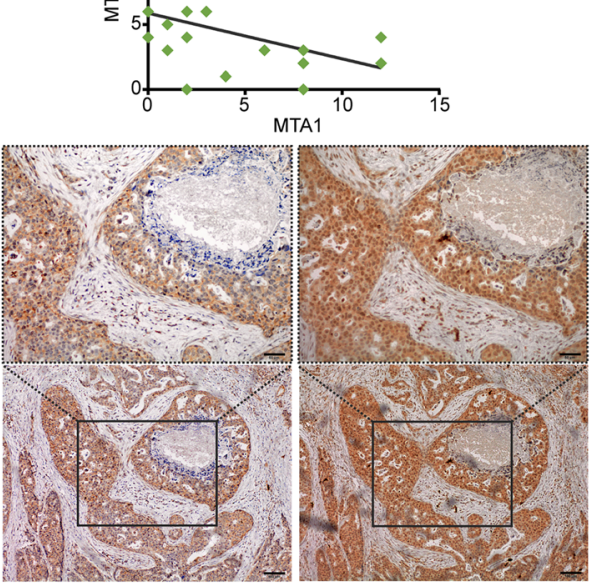

MTA1

MTA2

Fig. 2 (See legend on next page.) 
(See figure on previous page.)

Fig. 2 Overexpression of MTA1 downregulated MTA2 which promotes the metastasis of ZR-75-30 cells. a MTA2 was downregulated by MTA1 overexpression. b MTA2 was overexpressed in ZR-75-30-MTA2 cell line. c Overexpression of MTA2 significantly promoted the migration and (d) invasion of ZR-75-30 cells in vitro. 50,000 cells migrated for $20 \mathrm{~h} .40,000$ cells, invaded for $2.5 \mathrm{~d}$. Scale bars $=200 \mu \mathrm{m}, \mathrm{mean} \pm \mathrm{SD}, n=3$, ${ }^{* *} P<0.01$, ${ }^{* * *} P<0.001$, $t$ test. e MTA2 was knocked down by 2 shRNAs specifically targeting MTA2. $\mathbf{f}$ MTA2 knockdown significantly inhibited the migration and invasion of ZR-75-30 cells. 35,000 cells and 50,000 cells migrated for $36 \mathrm{~h}$ and invaded for $3.5 \mathrm{~d}$ respectively. Scale bars $=200 \mu \mathrm{m}, \mathrm{mean} \pm$ SD, $n=3,{ }^{* *} P<0.01,{ }^{* * *} P<0.001$, t test. $\mathbf{g}$ Overexpression of MTA1 did not repress the transcription of MTA2. Detected by $q R T-P C R$. Mean $\pm S D, n=3$, $R Q$ value: relative quantified value of mRNA expression. $\mathbf{h}$ The protein level of MTA1 and MTA2 are negatively correlated in tissues derived from luminal B breast cancer patients. Detected by IHC. Scale bars in upper box $=50 \mu \mathrm{m}$, scale bars in lower box $=100 \mu \mathrm{m}$. i Both overexpression of MTA1 and MTA2 increased EMT landmarks Snail and N-cadherin

pathway [22]. However, we did not detected the upregulation of MTA2 by immunoblotting (Fig. 3b). Further, we predicted enzymes that may cleave MTA2 by its amino acid sequence using the PROtease Specificity Prediction servER [23] (PROSPER). MTA2 was predicted to be cleaved by four types of proteases, including aspartic proteases, cysteine proteases, metalloproteases and serine proteases, among which the serine protease neutrophil elastase (NE) was predicted to cleave MTA2 with the highest probability (Fig. 3c). To evaluate whether NE can degrade MTA2, we overexpressed NE in ZR-75-30 cells, and as shown in Fig. 3d, the overexpression of NE led to a decrease in MTA2, and suprisingly, a decrease in MTA1.

To verify whether NE is responsible for MTA1-induced MTA2 degradation, we first treated ZR-7530-MTA1 cells with a specific NE inhibitor, sivelestat, with a concentration gradient or with the solvent of sivelestat (DMSO) as a control for $24 \mathrm{~h}$. As shown in Fig. 3e, the treatment of ZR-75-30-MTA1 cells with sivelestat restored MTA2 in a dose-dependent manner. Furthermore, we observed that the protein expression level of MTA1 also increased in the same manner as MTA2. The increase in MTA1 was not expected, but it further indicated that $\mathrm{NE}$ is responsible for MTA1-induced deregulation of MTA2 because the increase in MTA1 did not further downregulate MTA2. To further confirm the role of NE in MTA1-induced MTA2 degradation, we knocked down NE in ZR-75-30-MTA1 cells. The immunoblot results showed that knocking down NE led to a similar result as the sivelestat treatment (Fig. 3f).

As a serine protease that is stored in large quantities in neutrophil cytoplasmic azurophilic granules [24, 25], the interaction of NE with MTA2 that is located in the nucleus needed to be elucidated. Therefore, we cotransfected HA-tagged MTA2 with an empty vector or with FLAG-tagged NE into HeLa cells and analyzed the cellular location of these molecules by immunocytochemistry (ICC). HA-tagged MTA2 was exclusively detected in nuclei, while it was colocalized with NE in the cytoplasm (Fig. $3 g$ and $h$ ), providing evidence of an interaction between MTA2 and NE.
MTA1 repressed the transcription of elafin, an intrinsic NE inhibitor, in an HDAC- and/or DNMT-dependent manner To further exploit the association between MTA1 and serine protease NE, we tested whether MTA1 could transcriptionally regulate NE, its intrinsic inhibitors and some other intrinsic serine protease inhibitors. The qRT-PCR results showed that MTA1 overexpression significantly repressed the transcription of 2 intrinsic inhibitors of NE, elafin and $\alpha 1-\mathrm{PI}$. Besides, the transcription level of serine protease inhibitors Serpin B5 and Serpin B7 were also significantly decreased following MTA1 overexpression (Fig. 4a). We chose the dramatically decreased NE inhibitor, elafin, for further investigation. Immunoblotting also showed a decrease in elafin at the protein level in ZR-75-30-MTA1 cells (Fig. 4b). To further confirm that the downregulation of elafin by MTA1 leads to MTA2 degradation, we knocked down elafin in ZR-75-30 cells with two shRNAs and found a decrease in MTA2 and MTA1 expression levels, in accordance with the results shown in Fig. 3.

As a constitutive component of the NuRD complex, MTA1 could epigenetically modulate gene expression. To explore the mechanism by which MTA1 regulates elafin, we treated ZR-75-30-MTA1 cells with a concentration gradient of an HDAC inhibitor, suberoylanilide hydroxamic acid (SAHA, which is also known as vorinostat) and the solvent (DMSO) as a control for $48 \mathrm{~h}$. The qRT-PCR results showed that treatment with SAHA significantly upregulated elafin transcription in a dose-dependent manner, and the transcription level of elafin in ZR-75-30-MTA1 cells was even released to a level exceeded that of ZR-75-30-Vector cells after SAHA treatment, indicating a strong repression of elafin transcription by MTA1/HDACs (Fig. 4d). MTA1 and MBD2 (or MBD3) jointly participate in NuRD formation, and it has been reported that MTA1 forms a complex with DNMT to regulate gene expression. Therefore, we also hypothesized that MTA1 may regulate elafin transcription in a DNA methyltransferase (DNMT)-dependent manner. Treatment with a DNMT inhibitor, azacitidine (AZA), also led to a significant release of elafin transcription, indicating that MTA1 overexpression inhibited elafin transcription in a DNMT-dependent manner. 
a

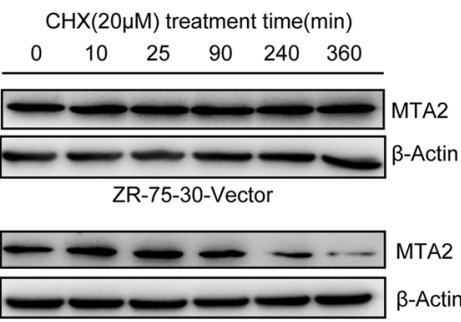

ZR-75-30-MTA1

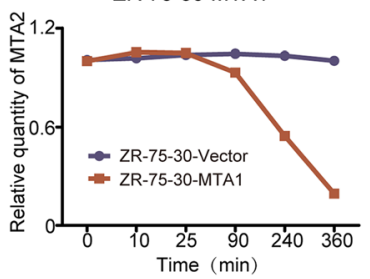

b
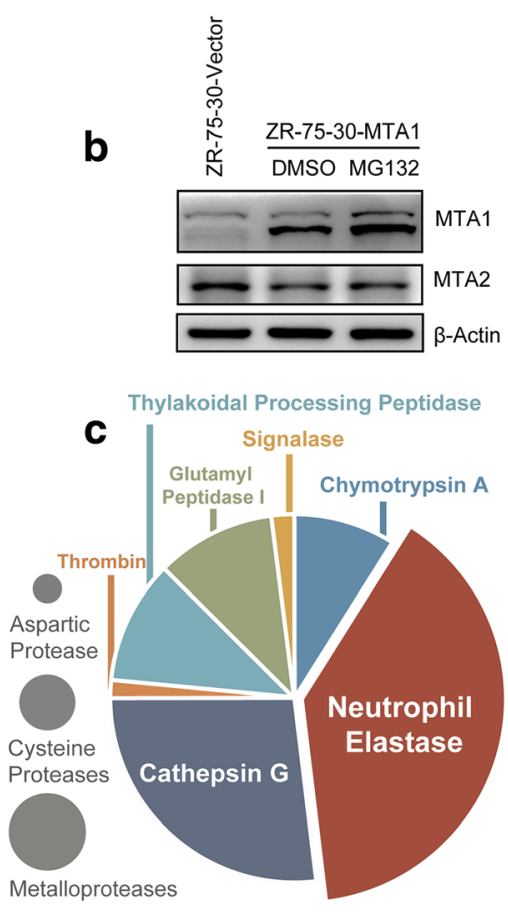

d
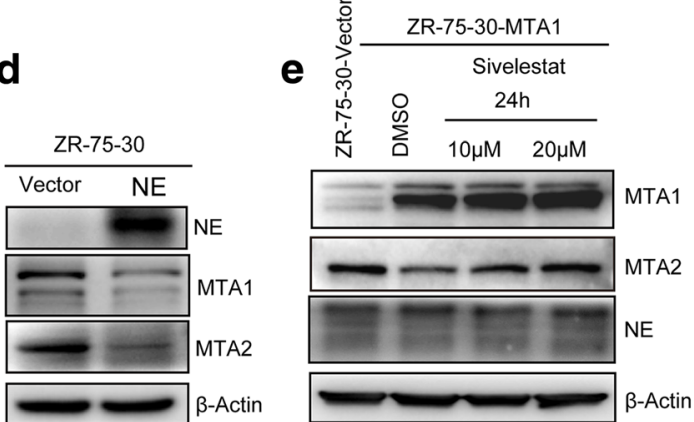

MTA1

\section{$\mathrm{E}$}

$\beta$-Actin
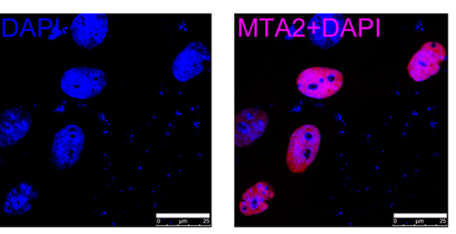

h
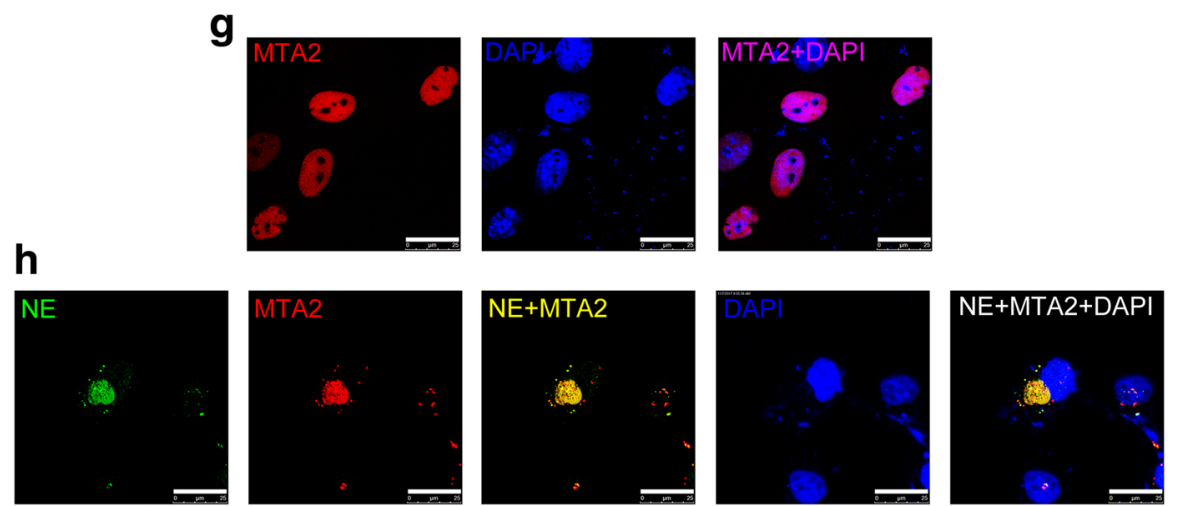

Fig. 3 NE is responsible for MTA1 induced MTA2 downregulation. a Overexpression of MTA1 decreased the protein stability of MTA2. b Treatment of ZR-75-30-MTA1 cells with MG132 (5 MM, 12 h) increased the protein level of MTA1 but did not increase that of MTA2. c Schematic diagram of the proteases predicted to cleave MTA2 at PROSPER website.NE is predicted to have the most cleavage sites in MTA2. $\mathbf{d}$ Overexpression of NE downregulated MTA2 and MTA1. e NE specific inhibitor sivelestat treatment restored MTA2, and upregulated MTA1, in ZR-75-30-MTA1 cells in a dose dependent manner. $\mathbf{f}$ NE knockdown increased the protein level of MTA2 and MTA1. g MTA2 located exclusively in nuclei. Detected by ICC. Scale bars $=25 \mu \mathrm{m}$. $\mathbf{h}$ MTA2 and NE co-localized in cytoplasm. Scale bars $=25 \mu \mathrm{m}$ 

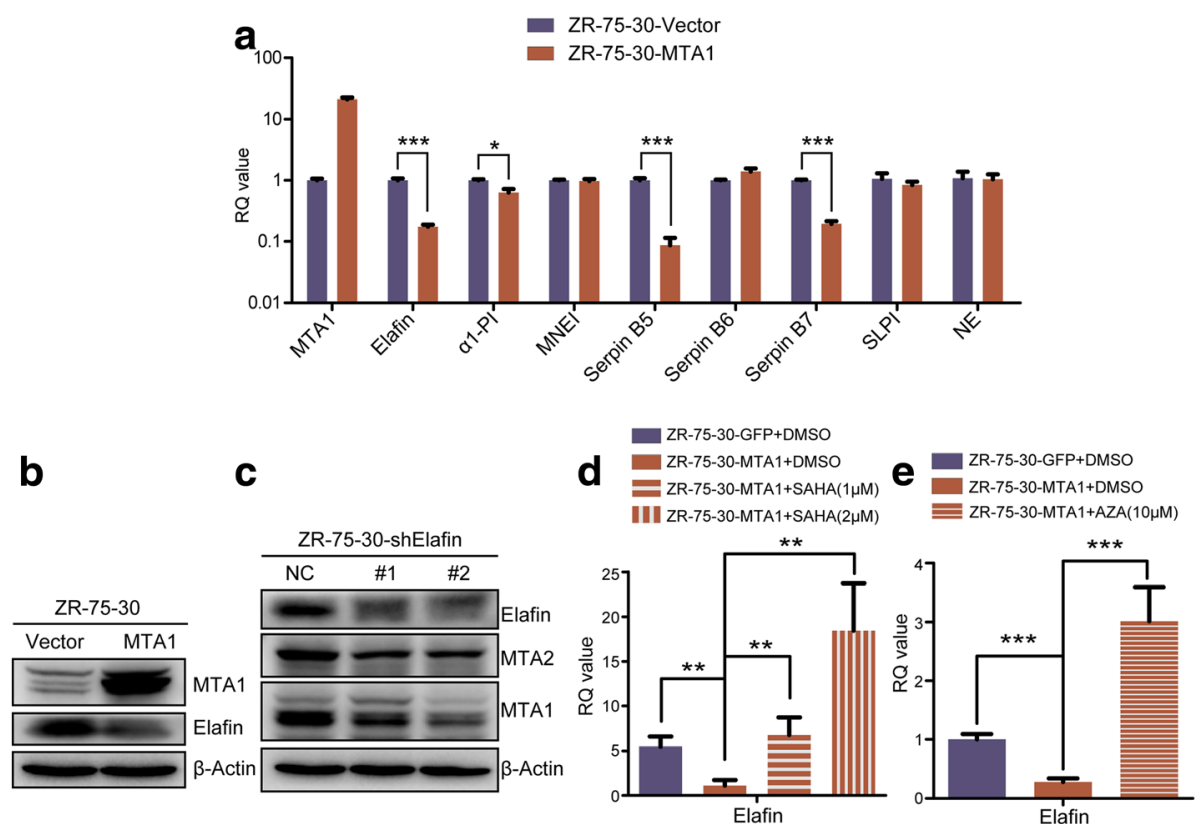

Fig. 4 MTA1 overexpression significantly inhibited the transcription of the intrinsic inhibitor of NE, elafin, in a HDAC and/or DNMT dependent manner. a Overexpression of MTA1 significantly downregulated the mRNA level of intrinsic inhibitors of NE, elafin and a1-PI, and serine protease inhibitors Serpin B5 and Serpin B7, but didn't significantly change the transcription of NE, MNEI, Serpin B6, and SLPI. Mean \pm SD, $n=3$, ${ }^{*} P<0.05$, ${ }^{* *} P<$ 0.001 , t test. $\mathbf{b}$ MTA1 overexpression decreased the protein level of elafin. $\mathbf{c}$ Knocking down of elafin decreased MTA2 and MTA1 at protein level. $\mathbf{d}$ HDAC inhibitor and e DNMT inhibitor treatments increased the mRNA level of elafin. ZR-75-30-MTA1 cells were treated with HDAC inhibitor SAHA and DNMT inhibitor, AZA, for $48 \mathrm{~h}$, after which the mRNA level of elafin significantly elevated, even to a value higher than that of ZR-75-30-Vector. Mean \pm $\mathrm{SD}, n=3,{ }^{* *} P<0.01,{ }^{* *} P<0.001$, t test

These results demonstrated that MTA1 overexpression led to the downregulation of elafin, an intrinsic inhibitor of NE, in an HDAC- and/or DNMT-dependent manner, thus impairing NE inhibition and promoting MTA2 degradation.

\section{NE cleaves MTA2 at its C-terminus}

To identify the NE cleavage site(s) in MTA2, we established a detection system in 293FT cells. We transiently transfected plasmid combinations pCMV-C-HA-MTA2 + Vector and pCMV-C-HA-MTA2 + pCMV-C-FLAG-MTA1 into 293FT cells and detected the protein and mRNA expression levels of MTA1-FLAG and MTA2-HA using antibodies against the corresponding tags and specific primers, respectively. In accordance with the observations in ZR-75-30 cells, the overexpression of MTA1-FLAG also decreased MTA2-HA expression at the protein level rather than at the mRNA level in 293FT cells (Fig. 5a and b).

Because NE can modulate the function of proteins by specific cleavage, we hypothesized that NE may cleave MTA2 at the functional domains, including the bromo-adjacent homology (BAH) domain (1-144 aa), Egl-27 and MTA1 homology 2 (ELM2) domain (145-256 aa), SANT domain (263-315 aa), zinc finger $(\mathrm{ZnF})$ domain (267-394 aa) and the C-terminus (395-668 aa) where its nuclear localization signals locate [26]. We constructed different C-terminal-truncated mutants of MTA2 fused with an HA tag that were designed based on the domains of MTA2, which were designated as MTA2-C-147-HA, MTA2-C-202-HA, MTA2-C-313-HA and MTA2-C-403HA (Fig. 5c), and cotransfected them with FLAG-tagged MTA1 or an empty vector as the control into 293FT cells. The immunoblot results showed that all $4 \mathrm{C}$-terminaltruncated mutants of MTA2 were downregulated by the co-expression of MTA1-FLAG (Fig. 5d).

The result shown in Fig. 5d indicated that MTA2 may be cleaved in a random pattern or may be cleaved at the C-terminus by NE. To validate this hypothesis, we constructed vectors containing different $\mathrm{N}$-terminal truncated mutants of MTA2 fused with an HA tag that were designed based on the domains of MTA2 (designated MTA2-N-201-HA and MTA2-N-312-HA) or the predicted cleavage sites of NE (designated MTA2-N-462-HA, MTA2-N-497-HA, MTA2-N-542-HA and MTA2-N-664HA) (Fig. 5e). We found that both MTA2-N-201-HA and MTA2-N-312-HA could not be downregulated by the co-expression of MTA1-FLAG (Fig. 5f). In addition, in other N-terminal truncated mutants, MTA2-N-462-HA could not be downregulated by the co-expression of MTA1; however, the MTA2-N-497-HA, MTA2-N-542HA and MTA2-N-664-HA mutants were downregulated when MTA1-FLAG was co-expressed (Fig. 5g). 


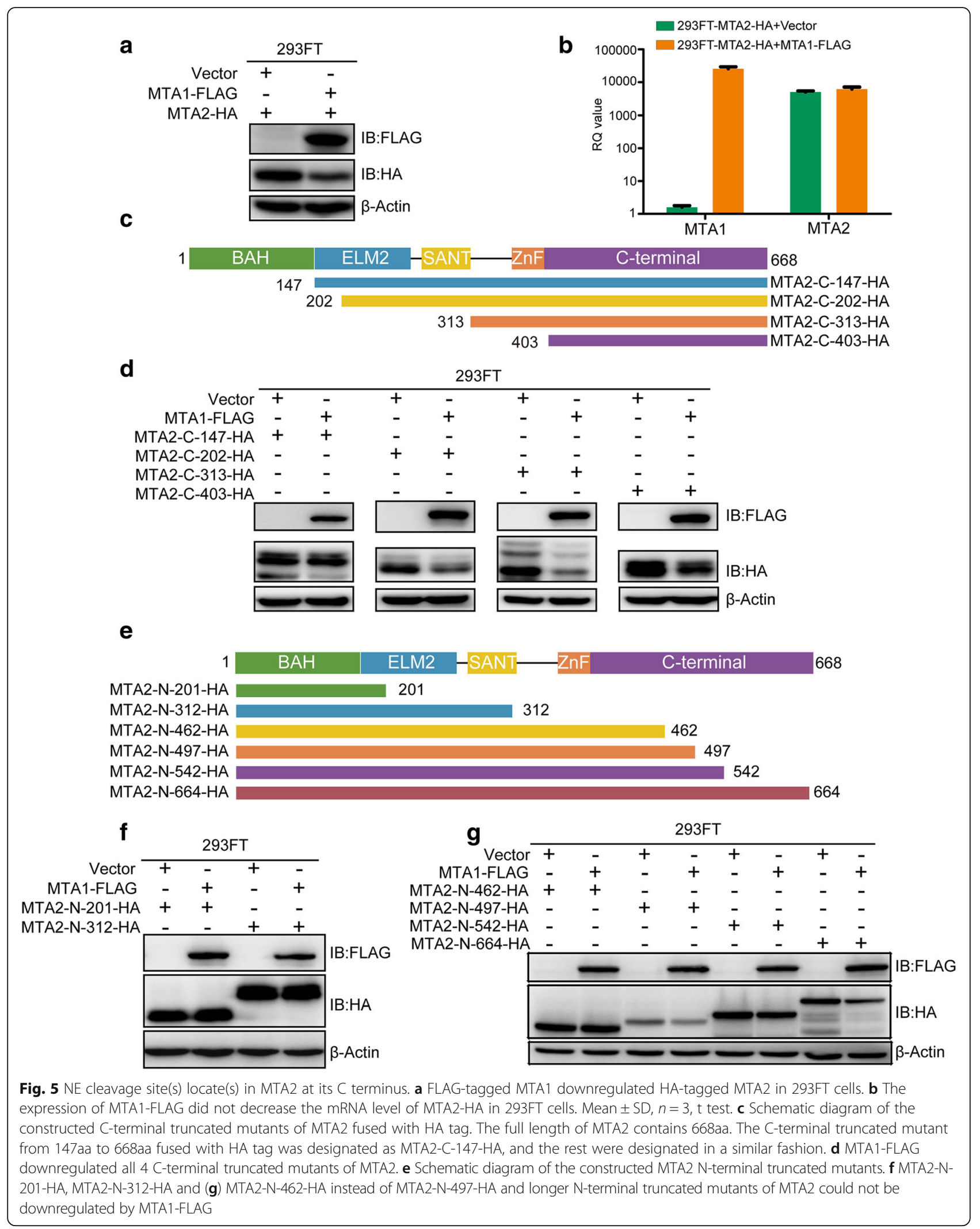


These results indicate that NE cleaves MTA2 at its C-terminus.

Identification of NE cleavage sites in the MTA2 C-terminus As predicted by the PROSPER website, there are 9 potential NE cleavage sites located in the MTA2
C-terminus, including the 462, 486, 497, 519, 542, 583, 621, 643 and 664 sites (Fig. 6a). To identify NE cleavage sites in the MTA2 C-terminus, we constructed different $\mathrm{N}$-terminal-truncated mutants either with or without point mutations to identify NE cleavage sites in the MTA2 C-terminus.

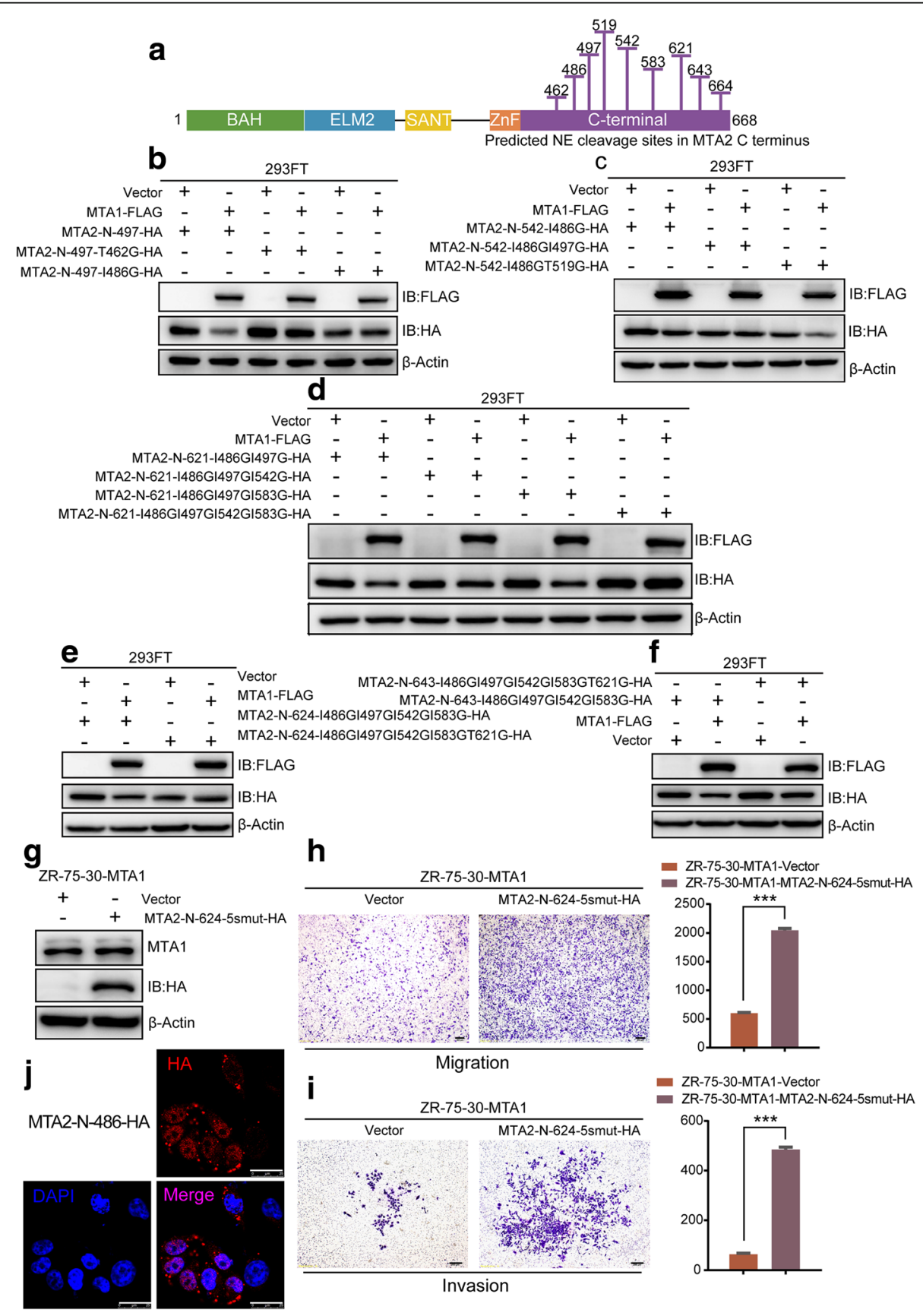

Fig. 6 486, 497, 542, 583 and 621 sites were identified to be NE cleavage sites of MTA2. a Schematic diagram of the predicted 9 NE cleavage sites after 461 locating in MTA2 C-terminus. b Identification of 486, (c) 497 (d) 542, 583 and (e) 621 as NE cleavage sites. f Unpredicted NE cleavage site(s) may locate between 621 and 643 sites. g MTA2-N-624-1486G/497GI542GI583GT621G-HA, designated as MTA2-N-624-5smut-HA was overexpressed in ZR-75-30 cells. $\mathbf{h}$ Overexpression of MTA2-N-624-5smut-HA significantly promoted the migration and (i) invasion of ZR-75-30MTA1 cells. Scale bars $=200 \mu \mathrm{m}$, mean $\pm S D, n=3$, ***P $<0.001$, $t$ test. $\mathbf{j}$ MTA2-N-486-HA showed a tendency of diffusing into cytoplasm. Scale bars $=25 \mu \mathrm{m}$ 
To validate whether 462 and/or 486 are/is NE cleavage site(s), we constructed an $\mathrm{N}$-terminal truncated mutant of MTA2, MTA2-N-497-HA, and mutated $462 \mathrm{~T}$ to 462G (MTA2-N-497-T462G-HA) and 486I to 486G (MTA2-N-497-I486G-HA), respectively. The immunoblot results showed that when co-expressed with MTA1-FLAG, MTA2-N-497-HA was downregulated. The I486G mutation, but not the T462G mutation, impeded the downregulation process (Fig. 6b). This result indicates that the 486 site is one of the NE cleavage sites in the MTA2 C-terminus, while the 462 site is not. Similar strategies were applied to identify that 497 (Fig. 6c), 542, 583 (Fig. 6d) and 621 (Fig. 6e) are NE cleavage sites.

However, the MTA2-N-643-I486GI497GI542GI583GT 621G-HA mutant was still downregulated by MTA1FLAG co-expression (Fig. 6f), suggesting that there might be unpredicted NE cleavage site(s) between site 621 and site 643 or that there might be another delicate and complex underlying mechanism; therefore, additional cleavage sites were not verified.

To elucidate whether restoring MTA2 would increase the migration and invasion capacity of ZR-75-30-MTA1 cells, we transfected plasmids containing MTA2-N-624I486GI497GI542GI583GT621G-HA (designated as MTA2$\mathrm{N}-624-5$ smut-HA thereafter) or empty vector as a control into ZR-75-30-MTA1 cells (Fig. 6g). In vitro migration and invasion assays indicated that overexpression of MTA2$\mathrm{N}-624-5$ smut-HA significantly promoted the migration and invasion of ZR-75-30-MTA1 cells (Fig. 6h and i).

To elucidate the co-localization of MTA2 and NE, we transfected plasmids expressing MTA2-N-486-HA into ZR-75-30 cells. The result of ICC detection indicated that the truncated MTA2 no longer showed a strong nucleus-located pattern as detected in Fig. 3g. It showed a tendency to diffuse into the cytoplasm (Fig. 6j). The result implies that $\mathrm{NE}$ cleavage at the $\mathrm{C}$-terminus destroyed the nuclear localization signals of MTA2 and led to the cytoplasm location of MTA2.

\section{Discussion}

As the first member of the MTA family, MTA1 has been linked to breast cancer metastasis since its discovery, and its crucial role in carcinogenesis and cancer metastasis has been revealed by important studies [7, 14, 15, 27-29]. Surprisingly, in our study, we found that MTA1 overexpression significantly inhibited the metastasis of ZR-75-30 luminal B breast cancer cells.

In accordance with previous reports, we found that overexpression of MTA1 promoted the migration and invasion of MDA-MB-231 cells in vitro. Besides, we found that both overexpression of MTA1 and MTA2 promoted EMT events in ZR-75-30 cells. However, both the migration and invasion of ZR-75-30 cells were impeded and promoted by MTA1 and MTA2 overexpression, respectively, showing the different roles MTA1 and MTA2 play in the metastasis of ZR-75-30 cells. The results are seemingly contradictory to the canonical roles of MTA1, but they are reasonable. It has been reported that MTA1 exhibited different expression and distribution patterns during the development of breast cancer in a mouse model, implying that the roles of MTA1 are altered sequentially [30]. Another member of MTA family, MTA3, which has been reported to inhibit the EMT of breast cancer by transcriptionally downregulating Snail [12], was reported to promote the progression of non-small cell lung cancer and hepatocellular carcinoma $[31,32]$, implying that the roles of MTA family members may change with different cancer types. Therefore, the inhibitory role of MTA1 in ZR-75-30 cells may be due to the specific context in this cell line or in luminal $\mathrm{B}$ breast cancer.

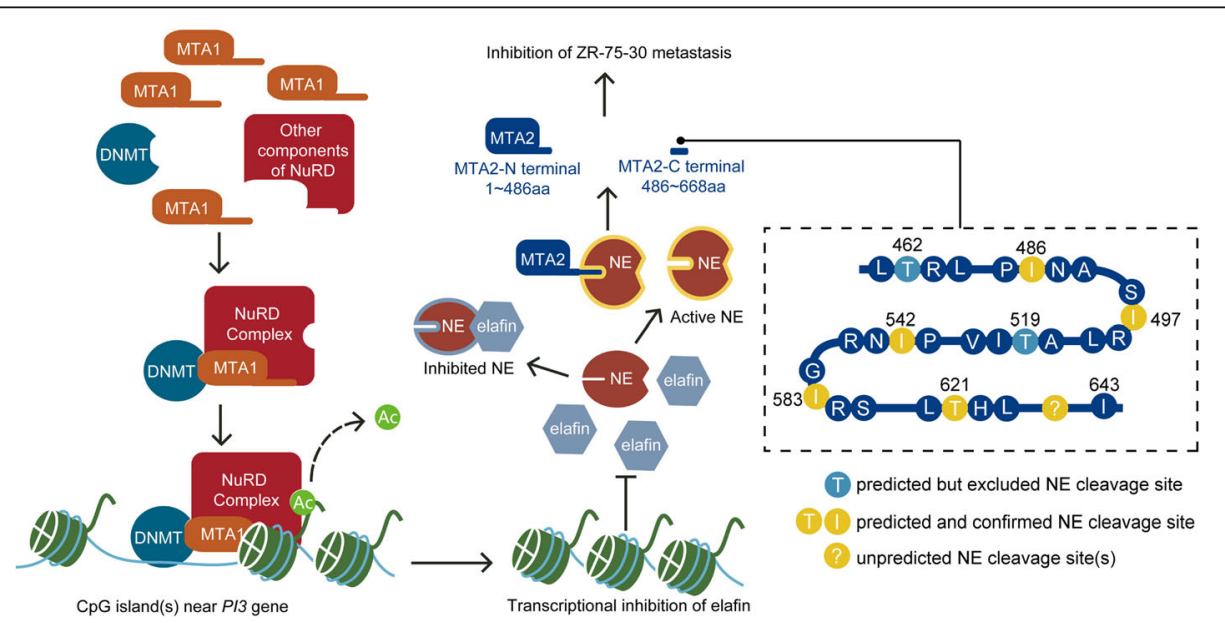

Fig. 7 Schematic diagram of the mechanism by which MTA1 downregulate MTA2 
We also reported that MTA1 overexpression posttranslationally, but not transcriptionally, downregulated MTA2 in ZR-75-30 cells. The same result has been reported in MCF-7 and T47D cells, but the mechanism has not been elucidated [19]. MTA2 shares $58 \%$ of its homology with MTA1 and exhibits functions that are similar to those of MTA1 [8]. In our study, both MTA1 and MTA2 evaluated the expression of Snail and $\mathrm{N}$-cadherin. Recently, it has been shown that EMT, as a stage of metastasis, can be divided into different transition states and endow different metastatic capacities to cancer cells, suggesting that enhanced EMT does not necessarily indicate enhanced metastasis [33]. Furthermore, it has also been reported that MTA2 can alter the activity of Rho GTPase by regulating Rho GDI $\alpha$ in MDA-MB-231 breast cancer cells [34]. This observation indicated that the different results of ZR-75-30 cell metastasis that we obtained in our study may be due to the different attributes of MTA1 and MTA2 in cytoskeleton modulation. Elucidating the difference between MTA1 and MTA2 may lead to an improved understanding of breast cancer metastasis.

NE is a serine protease that is mainly present in specialized lysosomes (azurophil granules) of neutrophils $[24,25]$. In breast cancer, it has been reported that NE can be secreted by breast cancer cells instead of normal breast cells, and a greater level of NE is associated with a more advanced stage and poor prognosis of breast cancer [35]. NE has also been reported to promote the metastasis of MDA-MB-231 breast cancer cells that can be inhibited by elafin [36]. In our study, we found that when co-expressed, NE and MTA2 were localized to the cytoplasm, suggesting that NE may cleave MTA2 in the cytoplasm. However, how co-expression of NE relocates MTA2 and exactly where they interact remains unknown. In addition, whether the interaction of NE with MTA2 is associated with its malignant roles in breast cancer and in what circumstances NE promotes or inhibits breast cancer metastasis remain interesting questions that require further exploration. In addition, beyond the downregulation of MTA2, we did not observe bands of the cleaved products of MTA2 by NE, which may suggest a more delicate and complex regulatory mechanism of MTA2 by NE.

To determine the link between NE and MTA2, we found that overexpression of MTA1 significantly inhibited the transcription of intrinsic inhibitors of NE, including $\alpha 1-\mathrm{PI}$ and elafin, and other serine protease inhibitors. MTA1 downregulated elafin in an HDACand DNMT-dependent/ NuRD complex-dependent manner. Elafin has been reported to inhibit the proliferation of breast cancer cells instead of normal cells [36], and during the tumorigenesis of breast cancer, elafin was shown to be downregulated in invasive breast cancer compared to normal mammary epithelium [37]. This result is consistent with the canonical role of MTA1 and is consistent with the results of our study. It can be speculated that at the initial stage of tumorigenesis, upregulated MTA1 downregulates elafin to promote breast cancer initiation, proliferation and progression. However, the roles of MTA1 and elafin seemingly change sequentially. Similar to the seemingly contradictory roles of MTA1, the residual expression of elafin in invasive breast cancer predicts a significantly poor prognosis of breast cancer patients: a tumor suppressor gene turned into an oncogene [37]. Our experimental results consistently indicated that the roles of oncogenes and anti-oncogenes, such as MTA1 and elafin, could be altered and that they could play different roles in different contexts.

\section{Conclusions}

Our study revealed the different roles that MTA1 and MTA2 play in ZR-75-30 metastasis and the mechanism underlying the downregulation of MTA2 by MTA1. MTA1 epigenetically inhibited the transcription of elafin, which decreased the inhibition of NE, and NE cleaved MTA2 in its C-terminus at the 486, 497, 542, 583, 621 sites to downregulate MTA2 posttranslationally, leading to the inhibition of the metastasis of ZR-75-30 cells in vitro (Fig. 7). These results provide an improved understanding of the difference and relationship between MTA1 and MTA2 and breast cancer metastasis as well as the flexible roles that genes may play in different contexts.

\section{Additional file}

Additional file 1: Table S1. Main primers used in the study. (DOC 64 kb)

\section{Abbreviations}

AZA: Azacitidine; BAH: Bromo-adjacent homology; CDS: Coding DNA sequence; CHX: Cycloheximide; DNMT: DNA methyltransferase; ELM2: Egl-27 and MTA1 homology 2; EMT: Epithelial-mesenchymal transition; FBS: Fetal bovine serum; HDAC: Histone deacetylase; HER2: Human epidermal growth factor receptor 2; ICC: Immunocytochemistry; MBD: Methyl-CpG-binding domain protein; MTA: Metastasis associated protein; NC: Negative control; NE: Neutrophil elastase; NuRD: Nucleosome remodeling and histone deacetylase; PROSPER: Protease specificity prediction server; qRT-PCR: Realtime quantitative reverse transcription polymerase chain reaction; $R Q$ value: Relative quantified value of mRNA expression; SAHA: Suberoylanilide hydroxamic acid; SANT: Swi3, Ada2, N-Cor, and TFIIIB; SDS-PAGE: Sodium dodecyl sulfate-polyacrylamide gel electrophoresis; TBST: Tris-buffered saline and Tween 20; ZnF: znic finger

\section{Acknowledgements}

We would like to show our special thanks to the support provided by Prof. Daqiang Li (Cancer Institute and Institute of Biomedical Sciences, Fudan University) to our study.

\section{Funding}

This study was supported by National Natural Science Foundation of China (81572827 and 91629301) to Weiguo Hu. 


\section{Availability of data and materials}

All experimental data generated or analyzed in this study are included in this article and its supplementary files.

\section{Authors' contributions}

LZ designed, performed the majority of the experiments, co-analyzed the data and co-wrote the manuscript. $L Z$, and $Y Z$ performed $I H C, Y Z$ and $W D$ performed IHC scoring and analysis. QO provided slices derived from luminal B patients and professional opinions. WD co-analyzed the data and co-wrote the manuscript. QW, YZ, JC, PD, LL, XZ, WZ, XL, LyL performed or participated in several experiments. JC, PZ and GC provided intellectual discussions and suggesstions. WH designed and directed the project. All authors read and approved the final manuscript.

\section{Ethics approval and consent to participate}

Not applicable

\section{Consent for publication}

Not applicable

\section{Competing interests}

The authors declare that they have no competing interests.

\section{Publisher's Note}

Springer Nature remains neutral with regard to jurisdictional claims in published maps and institutional affiliations.

\begin{abstract}
Author details
${ }^{1}$ Fudan University Shanghai Cancer Center and Institutes of Biomedical Sciences, Collaborative Innovation Center of Cancer Medicine, Shanghai Medical College, Fudan University, 270 Dong'an Road, Shanghai 200032, China. ${ }^{2}$ Department of Immunology, Shanghai Medical College, Fudan University, 130 Dong'an Road, Shanghai 200032, China. ${ }^{3}$ Cancer Institute, Fudan University Shanghai Cancer Center, Fudan University, 270 Dong'an Road, Shanghai 200032, China. ${ }^{4}$ Department of Colorectal Surgery, Fudan University Shanghai Cancer Center, 270 Dong'an Road, Shanghai 200032, China. ${ }^{5}$ Department of Oncology, Shanghai Medical College, Fudan University, 130 Dong'an Road, Shanghai 200032, China. ${ }^{6}$ Department of Breast Surgery, The Third Hospital of Nanchang, China Jiangxi Province Key Laboratory for Breast Diseases, 2 South Xiangshan Road, Nanchang 330009, Jiangxi, China.
\end{abstract}

\section{Received: 21 September 2018 Accepted: 2 January 2019} Published online: 14 January 2019

\section{References}

1. Siegel RL, Miller KD, Jemal A. Cancer statistics, 2018. CA Cancer J Clin. 2018; 68(1):7-30.

2. Eroles P, Bosch A, Perez-Fidalgo JA, Lluch A. Molecular biology in breast cancer: intrinsic subtypes and signaling pathways. Cancer Treat Rev. 2012; 38(6):698-707.

3. Bosch A, Eroles P, Zaragoza R, Vina JR, Lluch A. Triple-negative breast cancer: molecular features, pathogenesis, treatment and current lines of research. Cancer Treat Rev. 2010;36(3):206-15.

4. Kennecke H, Yerushalmi R, Woods R, Cheang MC, Voduc D, Speers CH, et al Metastatic behavior of breast cancer subtypes. J Clin Oncol. 2010;28(20): 3271-7.

5. Tran B, Bedard PL. Luminal-B breast cancer and novel therapeutic targets. Breast Cancer Res. 2011;13(6):221.

6. Fidler IJ. Critical factors in the biology of human cancer metastasis: twentyeighth G.H.a. Clowes memorial award lecture. Cancer Res. 1990;50(19):6130-8.

7. Toh Y, Pencil SD, Nicolson GL. A novel candidate metastasis-associated gene, mta1, differentially expressed in highly metastatic mammary adenocarcinoma cell lines. cDNA cloning, expression, and protein analyses. J Biol Chem. 1994;269(37):22958-63.

8. Futamura M, Nishimori H, Shiratsuchi T, Saji S, Nakamura Y, Tokino T. Molecular cloning, mapping, and characterization of a novel human gene, MTA1-L1, showing homology to a metastasis-associated gene, MTA1. J Hum Genet. 1999:44(1):52-6.
9. Simpson A, Uitto J, Rodeck U, Mahoney MG. Differential expression and subcellular distribution of the mouse metastasis-associated proteins Mta1 and Mta3. Gene. 2001;273(1):29-39.

10. Xue Y, Wong J, Moreno GT, Young MK, Cote J, Wang W. NURD, a novel complex with both ATP-dependent chromatin-remodeling and histone deacetylase activities. Mol Cell. 1998;2(6):851-61.

11. Zhang Y, Ng HH, Erdjument-Bromage H, Tempst P, Bird A, Reinberg D. Analysis of the NuRD subunits reveals a histone deacetylase core complex and a connection with DNA methylation. Genes Dev. 1999;13(15):1924-35.

12. Fujita N, Jaye DL, Kajita M, Geigerman C, Moreno CS, Wade PA. MTA3, a Mi2/NuRD complex subunit, regulates an invasive growth pathway in breast cancer. Cell. 2003;113(2):207-19.

13. Denslow SA, Wade PA. The human Mi-2/NuRD complex and gene regulation. Oncogene. 2007;26(37):5433-8.

14. Li DQ, Kumar R. Unravelling the complexity and functions of MTA Coregulators in human Cancer. Adv Cancer Res. 2015;127:1-47.

15. Pakala SB, Rayala SK, Wang RA, Ohshiro K, Mudvari P, Reddy SD, et al. MTA1 promotes STAT3 transcription and pulmonary metastasis in breast cancer. Cancer Res. 2013:73(12):3761-70.

16. Fu J, Qin L, He T, Qin J, Hong J, Wong J, et al. The TWIST/Mi2/NuRD protein complex and its essential role in cancer metastasis. Cell Res. 2011:21(2):275-89.

17. Hanahan D, Weinberg RA. Hallmarks of cancer: the next generation. Cell. 2011;144(5):646-74.

18. Thiery JP. Epithelial-mesenchymal transitions in tumour progression. Nat Rev Cancer. 2002;2(6):442-54.

19. Fujita N, Kajita M, Taysavang P, Wade PA. Hormonal regulation of metastasis-associated protein 3 transcription in breast cancer cells. Mol Endocrinol. 2004:18(12):2937-49.

20. Holliday DL, Speirs V. Choosing the right cell line for breast cancer research. Breast Cancer Res. 2011:13(4):215

21. Neve RM, Chin K, Fridlyand J, Yeh J, Baehner FL, Fevr T, et al. A collection of breast cancer cell lines for the study of functionally distinct cancer subtypes. Cancer Cell. 2006;10(6):515-27.

22. Li DQ, Ohshiro K, Reddy SD, Pakala SB, Lee MH, Zhang Y, et al. E3 ubiquitin ligase COP1 regulates the stability and functions of MTA1. Proc Natl Acad Sci U S A. 2009:106(41):17493-8.

23. Song J, Tan H, Perry AJ, Akutsu T, Webb Gl, Whisstock JC, et al. PROSPER: an integrated feature-based tool for predicting protease substrate cleavage sites. PLoS One. 2012;7(11):e50300.

24. Clark JM, Vaughan DW, Aiken BM, Kagan HM. Elastase-like enzymes in human neutrophils localized by ultrastructural cytochemistry. J Cell Biol. 1980;84(1):102-19.

25. Korkmaz B, Horwitz MS, Jenne DE, Gauthier F. Neutrophil elastase, proteinase 3, and cathepsin $\mathrm{G}$ as therapeutic targets in human diseases. Pharmacol Rev. 2010;62(4):726-59.

26. Wu M, Wang L, Li Q, Li J, Qin J, Wong J. The MTA family proteins as novel histone H3 binding proteins. Cell Biosci. 2013;3(1):1.

27. Ohshiro K, Rayala SK, Wigerup C, Pakala SB, Natha RS, Gururaj AE, et al. Acetylation-dependent oncogenic activity of metastasis-associated protein 1 co-regulator. EMBO Rep. 2010;11(9):691-7.

28. Nawa A, Nishimori K, Lin P, Maki Y, Moue K, Sawada H, et al. Tumor metastasis-associated human MTA1 gene: its deduced protein sequence, localization, and association with breast cancer cell proliferation using antisense phosphorothioate oligonucleotides. J Cell Biochem. 2000;79(2): 202-12.

29. Mazumdar A, Wang RA, Mishra SK, Adam L, Bagheri-Yarmand R, Mandal M, et al. Transcriptional repression of oestrogen receptor by metastasisassociated protein 1 corepressor. Nat Cell Biol. 2001;3(1):30-7.

30. Zhang H, Stephens LC, Kumar R. Metastasis tumor antigen family proteins during breast cancer progression and metastasis in a reliable mouse model for human breast cancer. Clin Cancer Res. 2006:12(5):1479-86.

31. Li H, Sun L, Xu Y, Li Z, Luo W, Tang Z, et al. Overexpression of MTA3 correlates with tumor progression in non-small cell lung Cancer. PLoS One. 2013:8(6):e66679.

32. Wang C, Li G, Li J, Li J, Li T, Yu J, et al. Overexpression of the metastasisassociated gene MTA3 correlates with tumor progression and poor prognosis in hepatocellular carcinoma. J Gastroenterol Hepatol. 2017;32(8): 1525-9.

33. Pastushenko I, Brisebarre A, Sifrim A, Fioramonti M, Revenco T, Boumahdi S, et al. Identification of the tumour transition states occurring during EMT. Nature. 2018;556(7702):463-8. 
34. Covington KR, Brusco L, Barone I, Tsimelzon A, Selever J, Corona-Rodriguez $A$, et al. Metastasis tumor-associated protein 2 enhances metastatic behavior and is associated with poor outcomes in estrogen receptornegative breast cancer. Breast Cancer Res Treat. 2013:141(3):375-84.

35. Yamashita Jl, Ogawa M, Ikei S, Omachi H, Yamashita SI, Saishoji T, et al. Production of immunoreactive polymorphonuclear leucocyte elastase in human breast cancer cells: possible role of polymorphonuclear leucocyte elastase in the progression of human breast cancer. Br J Cancer. 1994;69(1): 72-6.

36. Hunt KK, Wingate H, Yokota T, Liu Y, Mills GB, Zhang F, et al. Elafin, an inhibitor of elastase, is a prognostic indicator in breast cancer. Breast Cancer Res. 2013;15(1):R3

37. Caruso JA, Karakas C, Zhang J, Yi M, Albarracin C, Sahin A, et al. Elafin is downregulated during breast and ovarian tumorigenesis but its residual expression predicts recurrence. Breast Cancer Res. 2014;16(6):3417.

Ready to submit your research? Choose BMC and benefit from:

- fast, convenient online submission

- thorough peer review by experienced researchers in your field

- rapid publication on acceptance

- support for research data, including large and complex data types

- gold Open Access which fosters wider collaboration and increased citations

- maximum visibility for your research: over $100 \mathrm{M}$ website views per year

At BMC, research is always in progress.

Learn more biomedcentral.com/submissions 